\title{
Pneumoperitoneum After Percutaneous Endoscopic Gastrostomy: Does It Have Clinical Significance?
}

\author{
Ju Yup Lee, Kyung Sik Park \\ Department of Internal Medicine and Institute for Medical Science, Keimyung University School of Medicine, Daegu, Korea
}

\section{Article: Reappraisal of Pneumoperitoneum After Percutaneous Endoscopic Gastrostomy (Intest Res 2015;13:313-317)}

Since its first description in 1980 by Gauderer et al., ${ }^{1}$ percutaneous endoscopic gastrostomy (PEG) has become the method of choice for providing enteral access and nutritional support to patients who are unable to take oral feedings. ${ }^{2}$ The PEG-associated mortality rate is about $0.5 \%$ and various complications have been known to occur after PEG placement, including skin-site infection, acute hemorrhage, hematoma, aspiration, and perforation., Among these, unintentional perforation of the small bowel or colon is a major complication, and is associated with peritonitis and sepsis, which can be followed by septic shock and death if not diagnosed and treated promptly. ${ }^{3}$ Pneumoperitoneum detected on plain chest radiograph may be a sign of this serious complication. ${ }^{5}$ However, the air that is insufflated at the time of the PEG procedure by endoscopy and the air that enters the peritoneal cavity through a puncture site on the gastric wall can also develop into a pneumoperitoneum. ${ }^{3}$ Usually, most cases of pneumoperitoneum have a self-limited course, and clinicians regard a small pneumoperitoneum on radiographs as a benign finding of little clinical consequence.

In this issue of Intestinal Research, Park et al. retrospectively reviewed 193 patients who underwent PEG placement and reported that 9 (4.6\%) patients had a pneumoperitoneum on post-PEG radiographic imaging. ${ }^{6}$ Clinically signifi-

Received August 26, 2015. Revised August 31, 2015.

Accepted September 2, 2015

Correspondence to Kyung Sik Park, Department of Internal Medicine,

Dongsan Medical Center, 56 Dalseong-ro, Jung-gu, Daegu 41931, Korea. Tel:

+82-53-250-7088, Fax: +82-53-250-7442, E-mail: seenae99@dsmc.or.kr

Financial support: None. Conflict of interest: None. cant signs were observed in 5 (55.5\%) patients, with fever reported in 4, abdominal tenderness in 4 , and leukocytosis in 4 . Two patients $(22.2 \%)$ with moderate $(2-4 \mathrm{~cm})$ or large $(>4 \mathrm{~cm})$ pneumoperitoneum after PEG died from either pneumonia or septic shock. The authors concluded that the clinical course of pneumoperitoneum after PEG is not always benign and self-limited, and therefore a moderate or large pneumoperitoneum should not be ignored.

Although there may be some differences in PEG method and clinical environments, the incidence of pneumoperitoneum after PEG tube placement varies from $4.7 \%$ to $55.6 \%{ }^{6-9}$ However, cases of patients who experienced clinically significant signs of pneumoperitoneum such as fever, abdominal tenderness, or leukocytosis were rare in previous studies. ${ }^{5,8}$ Wiesen et al. reported that in the absence of clinical symptoms, post-PEG pneumoperitoneum is of no clinical significance, and does not warrant further intervention. ${ }^{2}$ In contrast, a retrospective study of 85 PEG patients reported that $5 \%$ of cases had evidence of pneumoperitoneum, including one insertion through the colon. ${ }^{3}$ The authors concluded that pneumoperitoneum, even without peritoneal signs and symptoms, may indicate iatrogenic bowel injury and requires follow-up and further diagnostic studies. ${ }^{3}$ Symptoms after PEG tube placement are important findings that suggest complicated pneumoperitoneum. However, the authors in the present study maintain that these clinically significant findings can be masked due to coexisting conditions, including the use of broad-spectrum antibiotics due to coexistent infection, altered mental status, and long-term sedation. Therefore, clinicians could have missed pneumoperitoneum

๑ Copyright 2015. Korean Association for the Study of Intestinal Diseases. All rights reserved.

This is an Open Access article distributed under the terms of the Creative Commons Attribution Non-Commercial License (http://creativecommons.org/licenses/by-nc/4.0)

which permits unrestricted non-commercial use, distribution, and reproduction in any medium, provided the original work is properly cited. 
in these situations. ${ }^{6}$

Several studies have showed conflicting results about the time to resolution as a predictive finding in complicated pneumoperitoneum. According to a study of PEG in intensive care patients, a large pneumoperitoneum that does not resolve within 72 hours can be an indication of complicated pneumoperitoneum. ${ }^{3}$ However, another study reported that a moderate or large pneumoperitoneum usually persists after 72 hours without any clinical symptoms. ${ }^{2}$ In the present study, the range of time to resolution of free air was 2-18 days, suggesting that time to resolution of pneumoperitoneum is a poor indicator for monitoring evidence of peritonitis.

In the present study, the authors used only the chest radiograph to detect pneumoperitoneum. Even with a small amount of air, the presence of pneumoperitoneum can easily be detected on chest radiograph according to most studies. ${ }^{2,3,6-9}$ Actually, however, CT is superior to simple chest radiograph in detecting the presence of pneumoperitoneum. ${ }^{10}$ In a retrospective study from the USA, CT revealed the presence of free air in patients who had no evidence of pneumoperitoneum on the chest radiograph. ${ }^{5}$ Therefore, when patients have peritonitis symptoms, clinicians should consider CT for the early detection of pneumoperitoneum.

Analysis of independent risk factors by comparing a postPEG pneumoperitoneum group with a non-pneumoperitoneum group may offer another important perspective. Unfortunately, in the present study, the authors did not perform this comparison and only showed the results for patients who developed pneumoperitoneum after PEG insertion. Furthermore, the retrospective study design is another limitation of the present study. The development of pneumoperitoneum can be influenced by various factors such as patient medical condition, underlying diseases, different operators, and where the PEG procedure was performed (intensive care unit or elsewhere). Further studies which consider these various biases will be necessary in the future.

In spite of the above limitations, the present study is a good reappraised of the clinical importance of pneumoperitoneum after PEG insertion. The clinical course is not always benign and self-limited. Peritoneal signs and symptoms may not be consistent with serious peritonitis, particularly in patients with altered mental status, or who received antibiotics or have prolonged sedation. Findings of a moderate or large pneumoperitoneum after PEG tube placement, even without peritoneal signs, should not be neglected, and should arouse suspicion of serious complicated pneumoperitoneum due to iatrogenic bowel injury.

\section{REFERENCES}

1. Gauderer MW, Ponsky JL, Izant RJ, Jr. Gastrostomy without laparotomy: a percutaneous endoscopic technique. J Pediatr Surg 1980;15:872-875.

2. Wiesen AJ, Sideridis K, Fernandes A, et al. True incidence and clinical significance of pneumoperitoneum after PEG placement: a prospective study. Gastrointest Endosc 2006;64:886889.

3. Milanchi S, Allins A. Early pneumoperitoneum after percutaneous endoscopic gastrostomy in intensive care patients: sign of possible bowel injury. Am J Crit Care 2007;16:132-136.

4. Gottfried EB, Plumser AB, Clair MR. Pneumoperitoneum following percutaneous endoscopic gastrostomy. A prospective study. Gastrointest Endosc 1986;32:397-399.

5. Dulabon GR, Abrams JE, Rutherford EJ. The incidence and significance of free air after percutaneous endoscopic gastrostomy. Am Surg 2002;68:590-593.

6. Park WY, Lee TH, Lee JS, et al. Reappraisal of pneumoperitoneum after percutaneous endoscopic gastrostomy. Intest Res 2015;13:313-317.

7. Wojtowycz MM, Arata JA, Jr., Micklos TJ, Miller FJ, Jr. CT findings after uncomplicated percutaneous gastrostomy. AJR Am J Roentgenol 1988;151:307-309.

8. Blum CA, Selander C, Ruddy JM, Leon S. The incidence and clinical significance of pneumoperitoneum after percutaneous endoscopic gastrostomy: a review of 722 cases. Am Surg 2009;75:39-43.

9. Alley JB, Corneille MG, Stewart RM, Dent DL. Pneumoperitoneum after percutaneous endoscopic gastrostomy in patients in the intensive care unit. Am Surg 2007;73:765-768.

10. Stapakis JC, Thickman D. Diagnosis of pneumoperitoneum: abdominal CT vs. upright chest film. J Comput Assist Tomogr 1992;16:713-716. 